\title{
Astrocyte-to-neurone lactate communication in the brain
}

\author{
A.G. Teschemacher*, B.V. Cardoso, V. Mosienko, B.H. Liu, S. Kasparov \\ University of Bristol, United Kingdom \\ *e-mail: anja.teschemacher@bristol.ac.uk
}
Key words: brain metabolism, central metabolic signalling, astrocytes, extracellular L-lactate, release of noradrenaline

\begin{abstract}
Astrocytes are thought to be the main source of extracellular L-lactate (LL) in the brain under physiological conditions. LL levels respond dynamically to neuronal network activity and metabolism. LL may serve as an additional energy substrate during periods of high activity. There is evidence that resting concentration of LL in astrocytes is higher than in neurones, allowing gradient-driven transfer of LL from astrocytes to neurones. This so called "lactate shuttle hypothesis" postulates that, under conditions of high energy demand, LL can be used by neurones as a preferred energy substrate. However, this idea is not universally accepted. Therefore, why astrocytes produce more LL than they can consume and whether the LL which astrocytes release is indeed used by neurones as fuel is not clear at present. In addition, increasing evidence indicates that LL has a signalling role in the brain. We demonstrated that LL released from astrocytes may release noradrenaline from noradrenergic neurones located in the brainstem and have been searching for the potential new receptor for LL in that area (Tang et al., Nature Communications, 2014). LL-mediated release of noradrenaline could be important for regulation of sleep/wake and attention states, learning and memory, and cardiorespiratory control.
\end{abstract}

However, almost all information we have about the physiological significance of astrocytic LL comes from acute experiments, and we currently lack the means for selective manipulation of LL release from astrocytes over longer time periods for further investigation of its actions in the brain of living animals. We therefore set out to develop an array of astrocyte-selective viral vectors that will allow us to specifically limit LL release by expressing enzymes which break down or decrease LL synthesis. Such enzymes can be 'borrowed' from bacteria. We show, using imaging, fluorimetry, and amperometry, that these constructs significantly decrease intracellular LL pool sizes. We anticipate that astrocyte-selective expression of these novel constructs will help to clarify the role of astrocytic LL in brain metabolism and central metabolic signalling. 